\title{
IL-17A-Blocker hemmt radiologische Progression bei PsA und AS
}

Die radiologische Progression einer Psoriasis-Arthritis (PsA) kann mit dem Interleukin (IL)-17A-Inhibitor Secukinumab gehemmt werden. Das belegen Studiendaten, die beim Jahreskongress des American College of Rheumatology in San Diego vorgestellt wurden [1]. Bei aktiver ankylosierender Spondylitis (AS) gibt es neue Langzeitdaten zu dem Biologikum: Bei den meisten Behandelten wurde über vier Jahre ein Stillstand der Wirbelsäulenverknöcherung festgestellt.

Rund 20 bis $30 \%$ aller PsoriasisPatienten entwickeln eine PsA, meist eine Polyarthritis, die mit einer schlechten Prognose verbunden ist, berichtete Professor Dr. Torsten Witte, Rheumatologe an der Medizinischen Hochschule Hannover. In der Regel würden im Therapieverlauf Biologika nötig. Eine Hemmung der radiologischen Progression bei PsA-Patienten, bisher nur für Tumornekrosefaktor (TNF)-alphaHemmer belegt, ist nach den neueren Daten auch mit Secukinumab möglich.

\section{Bei TNF-naiven Patienten war} Secukinumab besonders wirksam In der FUTURE-5-Studie wurden fast 1000 PsA-Patienten mit mehr als drei geschwollenen Gelenken, die unzureichend auf NSAR und/oder Methotrexat und/oder bis zu drei TNF-alpha-Blocker angesprochen hatten (Mease PJ et al., Arthritis Rheumatol 2017; 69(suppl10)), mit Secukinumab (Cosentyx ${ }^{\circledast}$,
150 mg oder 300 mg s. c.) oder Placebo behandelt. Sowohl bei anti-TNF-naiven (besonders deutlich) als auch-resistenten Patienten wurde mit dem IL-17ABlocker das ACR-20-Ansprechen deutlich verbessert, berichtete Witte (bei naiven Patienten bis zu 68\% nach 16 Wochen vs. $31 \%$ Placebo, $p<0,0001$ ).

\section{》) Rund 20-30\% aller Psoriasis-}

Patienten entwickeln eine PsA, meist eine Polyarthritis

Bei $88 \%$ der Behandelten in der Hochdosis-Gruppe kam es im Verlauf von 24 Wochen nicht zu einer radiologischen Progression (Veränderung des modifizierten Total Sharp Scores mTSS $\leq 0,5$ vs. $74 \%$ Placebo, $p<0,0001)$. Die strukturelle Progression von Gelenkschäden wurde deutlich gehemmt, betonte Witte. Sein Fazit: Die Wirkung von Secukinumab auf die radiologische Progression entspräche bei PsA-Patienten der

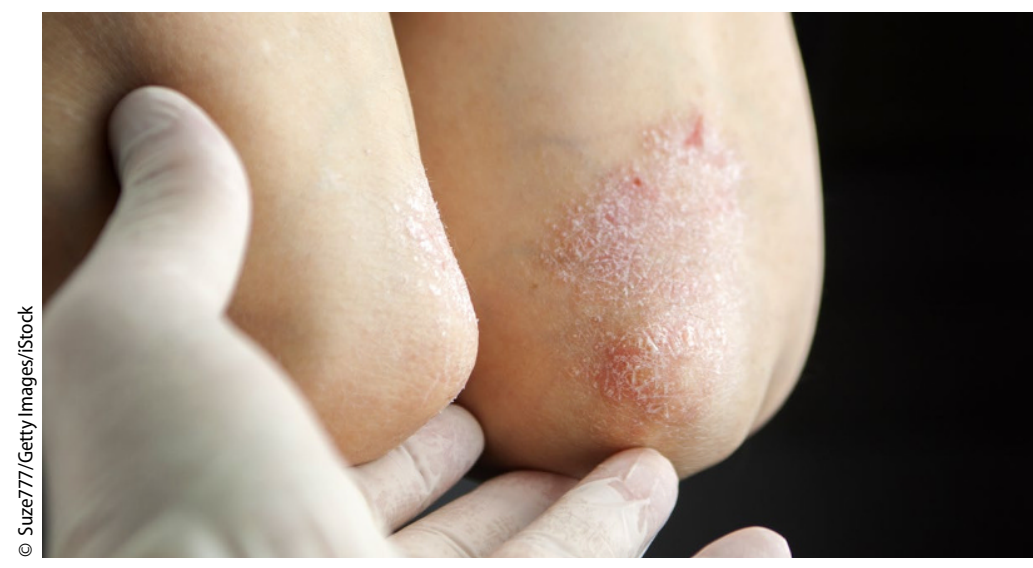

Abb. 1 A Etwa 20 -30\% aller Psoriasis-Patienten entwickeln auch eine Psoriasis-Arthritis von TNF-Blockern, vorteilhaft sei der IL-17A-Blocker für Hautbefunde und z. B. auch Enthesitis.

》) Secukinumab wirkt auf die radiologische Progression bei PsAPatienten ähnlich wie TNF-Blocker

Bei der AS-Therapie liegen inzwischen 4-Jahres-Daten für Secukinumab vor. Bei fast $80 \%$ der Behandelten wurde nach vier Jahren keine radiologische Progression an der Wirbelsäule (mSASS-Score <2) nachgewiesen, also kein Fortschreiten der Wirbelsäulenverknöcherung (Braun J et al., Arthritis Rheumatol 2017;69(suppl10). Auch die klinische Symptomatik besserte sich langanhaltend, berichtete Witte. In beiden Studien erwies sich die Therapie mit Secukinumab als sicher.

Roland Fath

\section{Literatur \\ 1. Mease PJ et al (2017) Subcutaneous Secukinumab Inhibits Radiographic Progression in Psoriatic Arthritis: Primary Results from a Large Randomized, Controlled, Double-Blind Phase 3 Study [abstract]. http://acrabstracts.org/abstract/ subcutaneous-secukinumab-inhibits-radio- graphic-progression-in-psoriatic-arthritis- primary-results-from-a-large-randomized- controlled-double-blind-phase-3-study/. Zugegriffen: 19. Jan. 2018}

rheuma plus $2018 \cdot 17: 16$ https://doi.org/10.1007/s12688018-0158-2

(c) Springer-Verlag GmbH Austria, ein Teil von Springer Nature 2018 